\title{
Non-invasive assessment of perfusion in acute stroke - reproducibility of BOLD delay measurements
}

Ahmed A Khalil1,2,3, Ayse Ceren Tanritanir ${ }^{1}$, Ulrike Grittner ${ }^{1,4}$, Arno Villringer ${ }^{2,3}$, Jochen B Fiebach ${ }^{1}$, Ralf Mekle $^{1}$

\author{
${ }^{1}$ Center for Stroke Research Berlin, Charité - Universitätsmedizin Berlin \\ ${ }^{2}$ Berlin School of Mind and Brain, Humboldt-Universität zu Berlin \\ ${ }^{3}$ Department of Neurology, Max Planck Institute for Human Cognitive and Brain Sciences, Leipzig \\ ${ }^{4}$ Department of Biostatistics and Clinical Epidemiology, Charité - Universitätsmedizin Berlin
}

\section{BACKGROUND}

- Blood flow assessment in acute ischemic stroke is important for identifying tissue at risk of progressing from reversible to irreversible damage and for monitoring treatment [1].

- Perfusion imaging techniques such as bolus-tracking MRI require intravenous administration of paramagnetic contrast agents. Non-invasive methods (such as arterial spin labeling) exist but are less reliable in patients with severe cerebrovascular disease [2].

- Measuring lags in low-frequency bloodoxygenation-level-dependent (BOLD) signal oscillations is an alternative noninvasive method for assessing blood flow [3]. This method, known as BOLD delay, reflects blood flow and has been validated in several settings $[4,5,6]$.

In this study, we investigated the reproducibility of BOLD delay measurements using a high temporal resolution MRI sequence in a cohort of acute stroke patients.

\section{METHODS}

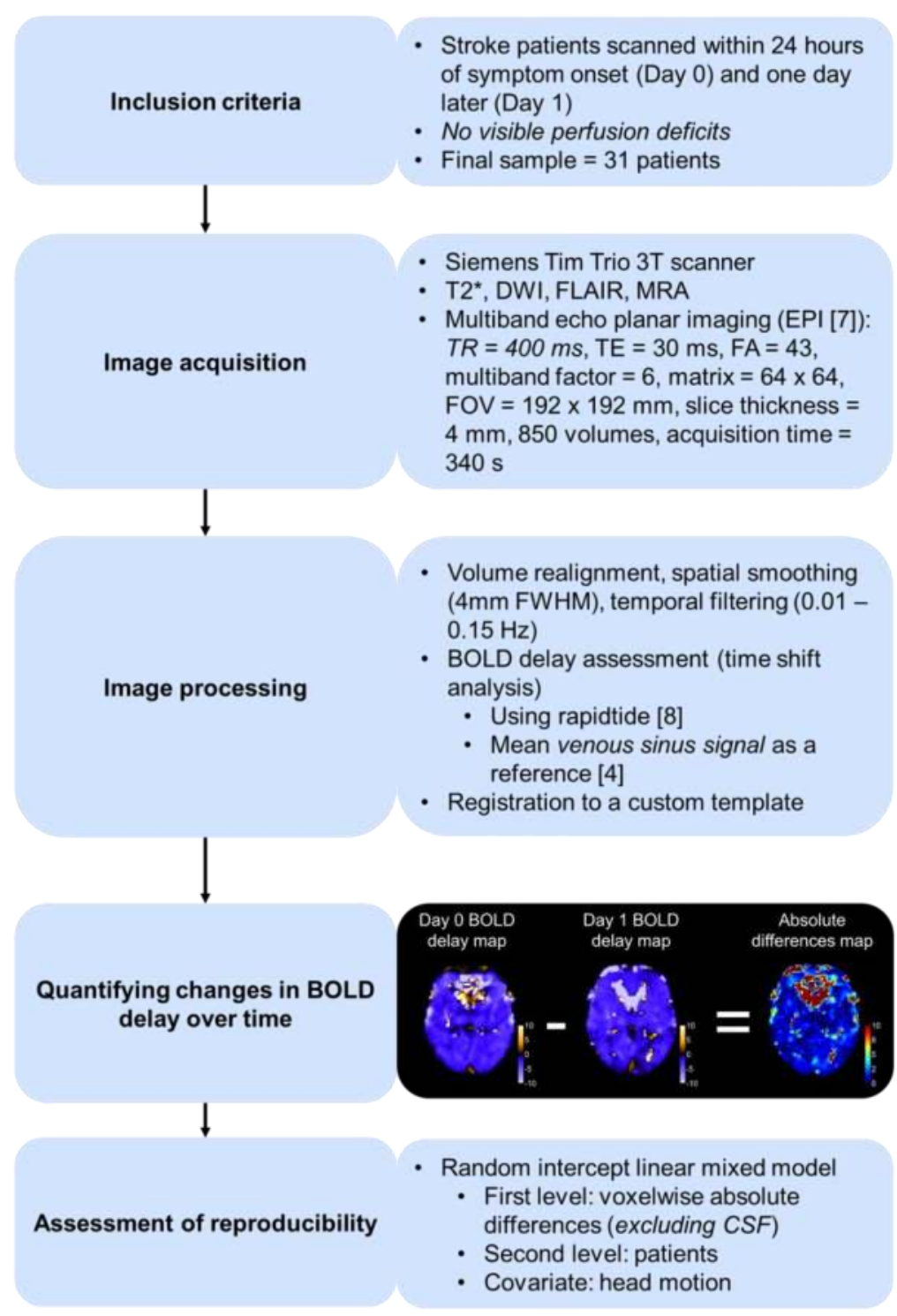

\section{RESULTS}

Clinical characteristics of the patients (median [IQR]):

- 24 males and 7 females

- Age $=73$ years [61 -78 years]

- Time from symptom onset to imaging $=8.8 \mathrm{~h}[3-11 \mathrm{~h}]$

- NIHSS $=1[1-3]$.

Mixed model results:

- Overall mean absolute BOLD delay difference $=3.0 \mathrm{~s}$ $(95 \% \mathrm{Cl}=1.5 \mathrm{~s}-4.5 \mathrm{~s}, \mathrm{t}=2.280)$.

- Head motion was positively associated with absolute differences in BOLD delay $(b=18.8,95 \% \mathrm{Cl}=8.8-$ $28.8, t=6.305)$ and the spread of these differences $(\rightarrow)$.
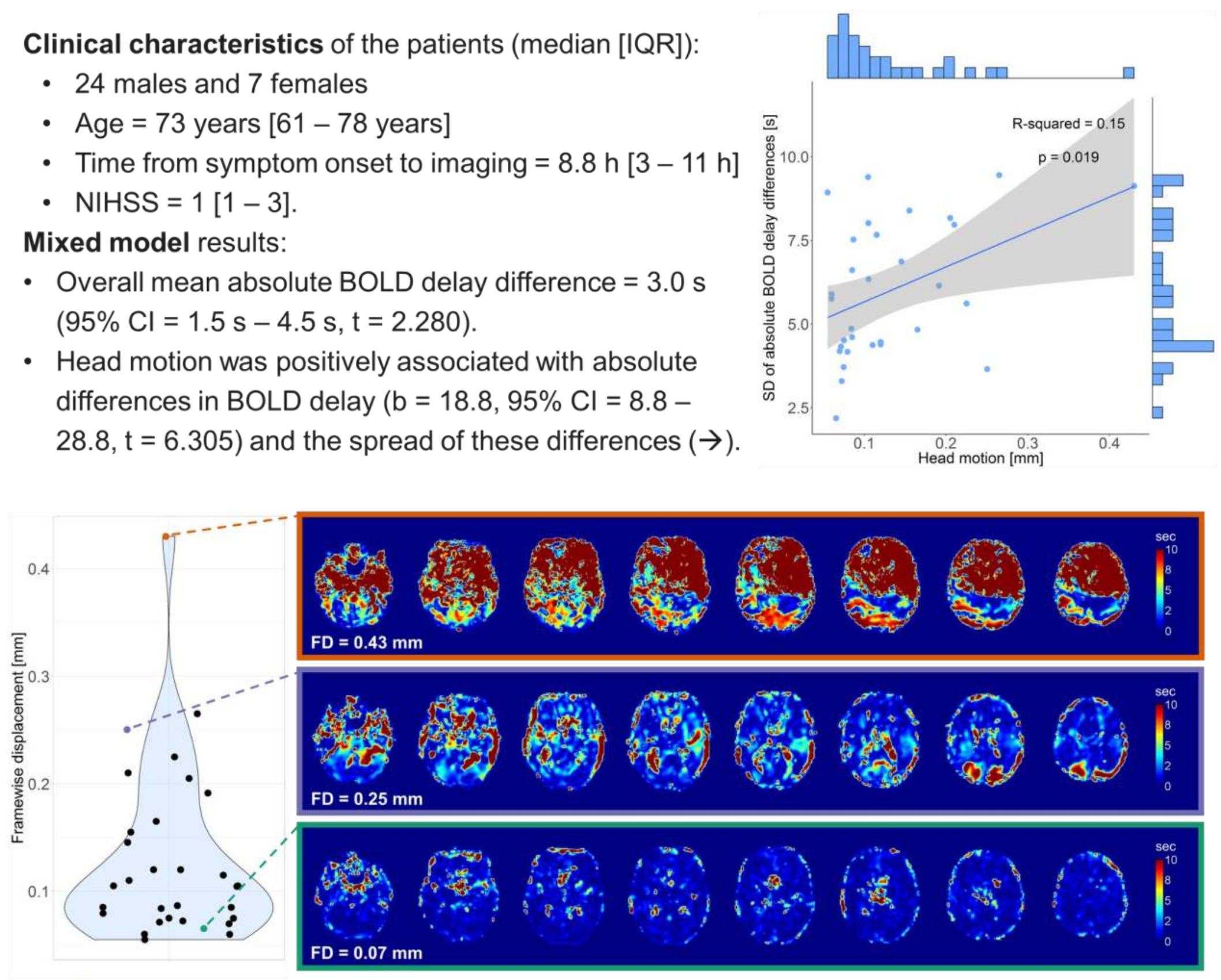

$\triangle$ Head motion was quantified using mean framewise displacement (FD) across all volumes [9] and averaged across Day 0 and Day 1 scanning sessions for each patient (shown for all patients in the violin plot to the left). Example absolute BOLD delay difference maps (in seconds) are shown on the right for three patients with varying degrees of head motion.

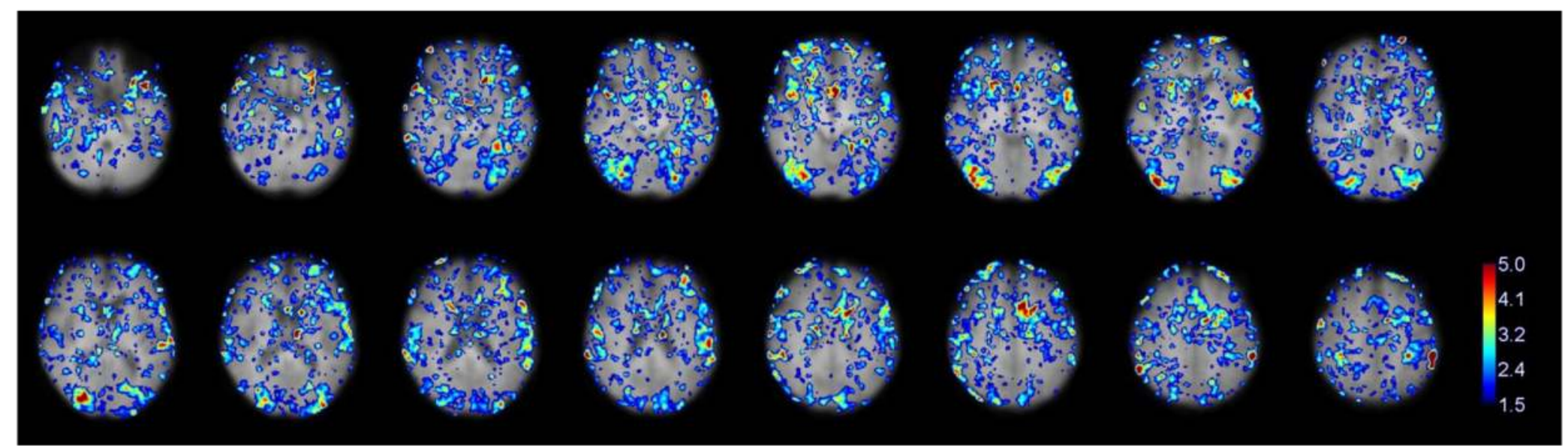

$\triangle$ Map showing the magnitude of absolute BOLD delay differences between timepoints (Day 0 and Day 1 ) in seconds, averaged across all patients. For better anatomical visualization, small differences $(<1.5 \mathrm{~s})$ are not shown in this figure. Large differences are seen prominently in the vasculature and the lateral occipital areas.

\section{CONCLUSIONS}

- In normally-perfused tissue, the magnitude of BOLD delay change over time is small compared to delays observed due to hypoperfusion in cerebrovascular diseases [4,5].

- Head motion adversely affects the reproducibility of BOLD delay.

- Physiological factors, such as cardiac or respiratory variations, may also contribute to the changes in BOLD delay over time observed in this study.

3

\author{
Acknowledgements: \\ Center for Magnetic Resonance Research (CMRR) \\ for kindly providing
used in this study. \\ ahmed-abdelrahim.khali@@charite.de
} Bukers et al, Stroke 2012 Khali, et al, Stroke 2017

Tong et al, JCBFM 2016 Xu et al, Neuroimage 2013

J] Frederick, B, rapidtide [Computer Software] (2016). doi:10.5281/zenodo.814991
Power et al, Neuroimage 2012 